\title{
Fully automated screening of veterinary drugs in milk by turbulent flow chromatography and tandem mass spectrometry
}

\author{
Alida A. M. Stolker • Ruud J. B. Peters • \\ Richard Zuiderent • Joseph M. DiBussolo • \\ Cláudia P. B. Martins
}

Received: 30 December 2009/Revised: 12 March 2010/Accepted: 15 March 2010/Published online: 9 April 2010

(C) The Author(s) 2010. This article is published with open access at Springerlink.com

\begin{abstract}
There is an increasing interest in screening methods for quick and sensitive analysis of various classes of veterinary drugs with limited sample pre-treatment. Turbulent flow chromatography in combination with tandem mass spectrometry has been applied for the first time as an efficient screening method in routine analysis of milk samples. Eight veterinary drugs, belonging to seven different classes were selected for this study. After developing and optimising the method, parameters such as linearity, repeatability, matrix effects and carry-over were studied. The screening method was then tested in the routine analysis of 12 raw milk samples. Even without internal standards, the linearity of the method was found to be good in the concentration range of 50 to $500 \mu \mathrm{g} / \mathrm{L}$. Regarding repeatability, RSDs below $12 \%$ were obtained for all analytes, with only a few exceptions. The limits of
\end{abstract}

A. A. M. Stolker · R. J. B. Peters

RIKILT Institute of Food Safety,

P.O. Box 230, 6700 AE Wageningen, The Netherlands

R. Zuiderent

Thermo Fisher Scientific,

Takkebijsters 14817 BL Breda, The Netherlands

J. M. DiBussolo

Chemistry Department, Thermo Fisher Scientific's Applications

Laboratory at West Chester University of Pennsylvania,

750 S. Church Street,

West Chester, PA 19383, USA

C. P. B. Martins $(\square)$

Thermo Fisher Scientific,

Acero 30-32, Planta. 2, Mod. 3,

08038 Barcelona, Spain

e-mail: claudia.martins@thermofisher.com detection were between 0.1 and $5.2 \mu \mathrm{g} / \mathrm{L}$, far below the maximum residue levels for milk set by the EU regulations. While matrix effects-ion suppression or enhancementare obtained for all the analytes the method has proved to be useful for screening purposes because of its sensitivity, linearity and repeatability. Furthermore, when performing the routine analysis of the raw milk samples, no false positive or negative results were obtained.

Keywords Veterinary drugs · Milk · Turbulent flow Screening $\cdot$ Tandem mass spectrometry

\section{Introduction}

Incorrect use of drugs in veterinary practice may leave residues in edible tissues that can have direct toxic-effects on consumers. The EU has set maximum residue limits (MRLs) for a variety of veterinary drugs in tissues, milk and eggs $[1,2]$. In order to detect such residues in food and tissues, microbiological or bioassay techniques are widely used as screening methods. These methods generally do not distinguish between members of a class of antibiotics, but provide a semi-quantitative estimate of 'total' residues detected. Nevertheless, they continue to be used because of their simplicity and low cost.

The cost-effectiveness of analytical procedures is becoming an important issue for all laboratories involved in residue analysis. A way to improve cost-effectiveness is to maximise the number of analytes that may be determined by a single procedure or from a single portion of test material, and/or to limit the sample pre-treatment steps. Many analytical methods for the determination of residues of veterinary drugs using different extraction procedures, 
clean-up conditions and detection principles are available. In general, a liquid-liquid extraction (LLE) is performed followed by an extract clean-up and concentration step using solid-phase extraction (SPE), separation and detection by liquid chromatography tandem mass spectrometry (LC-MS/MS) [3]. Furthermore, most of the procedures described are dealing with one group of analytes or one class of veterinary drugs.

Recently, some multi-residue methods for the analysis of drugs in milk have been published. Turnipseed et al. [4] described a method for the analysis of different classes of veterinary drugs in milk using LLE followed by SPE and LC-MS/MS. Gaugain-Juhel et al [5] monitored 58 analytes in milk using a simple LLE followed by LC-MS/MS. However, not all classes of veterinary drugs were tested on the described studies. Instead, only antibiotic compounds have been used. Aguilera-Luiz et al. [6] reported an interesting approach using the so called QuEChERS (quick, easy, cheap, effective, rugged and safe) extraction technique. This extraction although relatively quick still included several sample handling steps like LLE, centrifugation, addition of an adsorbent, centrifugation, dilution and others.

Recently, Kinsella et al. presented a comprehensive review on the current trends in sample preparation for the isolation of veterinary drugs and growth promoters from foods. Turbulent flow chromatography coupled with tandem mass spectrometry (TFC-LC-MS/MS) was described as a technique that eliminates time-consuming sample clean-up, increases productivity and reduces solvent consumption without sacrificing sensitivity or productivity [7].

TFC was introduced in the late 1990s as a technique for the direct injection of biological fluids onto a column packed with $50 \mu \mathrm{m}$ spherical porous particles [8, 9]. Nowadays, TFC can be used as a high-throughput sample preparation technique that makes use of high flow rates in 0.5 or $1.0 \mathrm{~mm}$ internal diameter columns packed with particles in the size range of $30-60 \mu \mathrm{m}$. Due to these large particle sizes, there is only moderate back-pressure on the column. Samples are applied to the column using an aqueous mobile phase as illustrated in Fig. 1 [10]. Small molecules diffuse more extensively than macromolecules (e.g., proteins, lipids, sugars) and are driven into the pores of the sorbent. Due to the high flow rate, the larger molecules and matrix constituents are flushed to waste and do not have an opportunity to diffuse into the particle pores. The trapped analytes are desorbed from the TFC column by back-flushing it with an organic solvent and the eluate can be transferred with a switching valve onto the analytical LC-MS/MS system for further separation and detection. Although TFC is a promising technique for rapid sample preparation, very few uses in residue analysis have been reported in the literature. Mottier et al. carried out quantitative analysis of 16 quinolones in honey using TFC coupled on-line to LC-MS/MS [11]. Sample preparation involved simple dilution with water followed by filtration and transfer of an aliquot into a vial. Sample extraction time was $4.5 \mathrm{~min}$, while the overall analysis took $18.5 \mathrm{~min}$. Recovery of the method ranged from $85 \%$ to $127 \%$, while the LOD of the method was $5 \mu \mathrm{g} / \mathrm{kg}$. Krebber et al. used TFC-LC-MS/MS for the rapid determination of enrofloxacin and ciprofloxacin in edible tissues [12]. Tissue samples (bovine, porcine, turkey, rabbit) were extracted with acetonitrile:water:formic acid, filtered and an aliquot injected onto the TFC-LC-MS/MS system. The run time for the analysis was $4 \mathrm{~min}$. The LOQ of the method was $25 \mu \mathrm{g} / \mathrm{kg}$ in all matrices. The recovery of the method ranged from $72 \%$ to $105 \%$. So far, TFC is only used for the analysis of compound of one specific class of veterinary drugs. In this study TFC-LC-MS/MS is applied to the analysis of residues of multi-class antibiotics in milk.

The selected veterinary drugs were the benzimidazole: albendazole, the sulphonamide: sulphamethazine, the nonsteroidal anti-inflammatory agent (NSAID): phenylbuta-
Fig. 1 Schematic representation of the loading step in an Aria TLX-2 system. The sample is introduced into the system by an autosampler and a loading pump. The system also involves a solvent holding loop connected to valve A. This loop contains a solvent mixture strong enough to elute the analytes from the TurboFlow column (TFC) into the analytical column (transfer step). An eluting pump delivers a mixture of solvents and enables a normal chromatographic separation and detection

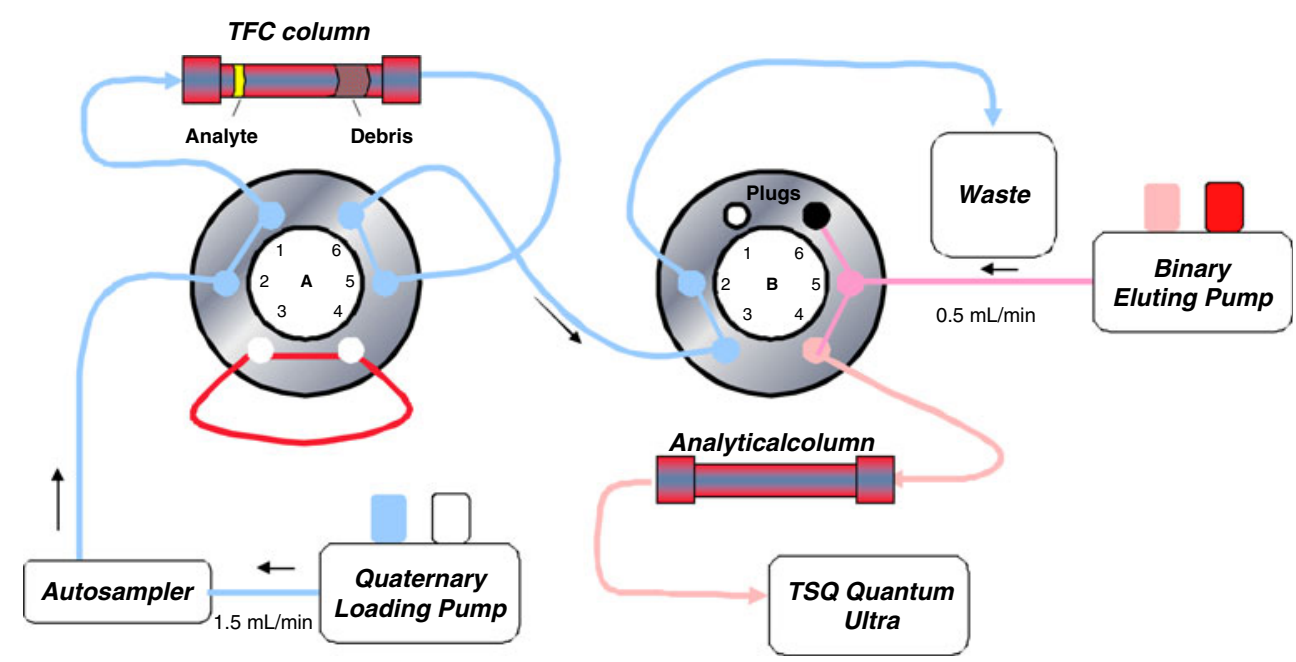


zone, the quinolone: difloxacin, the macrolide: spiramycin, the tetracyclines: tetracycline and oxytetracycline and the ionofore: salinomycin-Na. Milk was selected as the test matrix because it is an important matrix for residue control which can contain residues from different classes of veterinary drugs. Different milk samples, containing different fat percentages were studied. Finally, the method has been tested in a routine environment, where 12 raw milk samples were analysed and compared with the data obtained when analysing the same batch of samples by a different method. The results of method development and application will be described and discussed.

\section{Materials}

Chemicals, reagents and solutions

Methanol, acetonitrile, ammonium acetate, sodium EDTA, formic acid, trifluoroacetic acid, propanol and acetone were obtained from Biosolve (Valkenswaard, the Netherlands).

The analytical standards albendazole, pheylbutazone, spiramycin, tetracycline, oxytetracycline and salinomycin$\mathrm{Na}$ were purchased from Sigma-Aldrich Chemie b.v (Zwijndrecht, the Netherlands). The analytical standards of sulphamethazine (sulphadimidine) and difloxacin were purchased from Riedel de Haen (Seelze, Germany).

A diluent consisting of: $50 \mathrm{mM}$ ammonium acetate + $7.5 \mathrm{mM}$ sodium EDTA in water/acetonitrile $(1 / 1 ; v / v)$ was prepared to induce protein precipitation.

Individual veterinary drug stock solutions $(0.1,1$ or $2 \mathrm{mg} / \mathrm{ml})$ were prepared in pure methanol and stored at $-18^{\circ} \mathrm{C}$. The mixed standard solution containing all eight veterinary drugs was prepared in diluent, stored at $-18^{\circ} \mathrm{C}$ and used for 1 week.

\section{Samples}

Whole (fat content approximately 3\%), skimmed (fat content $<0.5 \%$ ) and semi-skimmed (fat content $1.5-2 \%$ ) milk samples were used for method development. These test samples were obtained from a local supermarket in Pennsylvania, USA.

All milk samples used for the application test were raw (not pasteurised) milk samples. The samples were collected by the Dutch Food and Consumer Product Safety Authority, Laboratory Region East (Wageningen, The Netherlands) at farmhouses during the spring of 2009. The samples were received in frozen conditions and were kept frozen $\left(-18^{\circ} \mathrm{C}\right)$ until analysis.

\section{Equipment}

A turbulent flow chromatograph Aria TLX-2 system (Thermo Fisher Scientific, Franklin, MA) was used for these experiments and was comprised of a PAL autosampler (CTC
Analytics, Zwingen, Switzerland), a low-pressure mixing quaternary pump (loading pump), a high-pressure mixing binary pump (eluting pump) and a three-valve switching device unit with six-port valve. The entire system was controlled via Aria software, version 1.6. The TurboFlow TFC columns used were connected in tandem: Cyclone, $50 \times 0.5 \mathrm{~mm}, 60 \mu \mathrm{m}$ particle size, $60 \AA$ pore size and Cyclone $\mathrm{P} 50 \times 0.5 \mathrm{~mm}, 60 \mu \mathrm{m}$ particle size, $60 \AA$ pore size (Thermo Fisher Scientific, Franklin, MA); whereas the analytical HPLC column was a Betasil Phenyl Hexyl column 3.0 $\times 50,3 \mu \mathrm{m}$; Thermo Fisher Scientific, Runcorn, UK. The temperature of the analytical column was maintained at $50^{\circ} \mathrm{C}$ using a column heater.

The triple quadrupole mass spectrometer was a TSQ Quantum Ultra (Thermo Fisher Scientific, San Jose, CA) equipped with a heated electrospray ionisation probe that was kept at $475^{\circ} \mathrm{C}$. All analyses were performed in the positive ionisation mode with a spray voltage set at $3,500 \mathrm{~V}$. The sheath, auxiliary and ion sweep gas used was nitrogen at 50 , 25 and 2 arbitrary units, respectively. The system was operated in the selected reaction monitoring (SRM) mode with argon as the collision gas at a pressure of $1.5 \mathrm{mTorr}$. The ion transfer tube was kept at $250^{\circ} \mathrm{C}$ while the scan time and width were $0.10 \mathrm{~s}$ and $0.02 \mathrm{~m} / \mathrm{z}$, respectively. Data were processed using LCQuan software version 2.6.

\section{Methods}

Sample preparation

Protein precipitation was induced by mixing $100 \mu \mathrm{L}$ of milk sample with $900 \mu \mathrm{L}$ of diluent. After centrifugation of the mixture at $10,000 \mathrm{rpm}$ for $10 \mathrm{~min}$, the supernatant was collected and $50 \mu \mathrm{L}$ analysed by TFC-LC-MS/MS.

\section{Chromatographic conditions}

The gradient used is illustrated in Table 1. The solvent system used was as follows:

Loading pump A: $0.1 \%$ formic acid and $0.05 \%$ trifluoroacetic acid in water; $\mathrm{B}$ : methanol; $\mathrm{C}$ : isopropanol: acetone $(1 / 1, v / v) ; \mathrm{D}: 2 \%$ acetonitrile and $0.1 \%$ ammonium hydroxide in water.

Eluting pump A: $0.1 \%$ formic acid and $0.01 \%$ trifluoroacetic acid in water; B: methanol.

\section{Detection}

The TFC-LC-MS/MS analysis was carried out using the conditions stated in the "Equipment" section. The optimi- 
Table 1 Gradient used on the loading pump and eluting pump for the TFC-LC (ESI)-MS/MS analysis of the milk samples
Loading pump-A: $0.1 \%$ formic acid and $0.05 \%$ trifluoroacetic acid in water; $\mathrm{B}$ : methanol; $\mathrm{C}$ : isopropanol:acetone $(1 / 1, v / v)$; D: $2 \%$ acetonitrile and $0.1 \%$ ammonium hydroxide in water

Eluting pump-A: $0.1 \%$ formic acid and $0.01 \%$ trifluoroacetic acid in water; B: methanol

\begin{tabular}{lccccccccc}
\hline & \multicolumn{3}{c}{ Loading pump } & & \multicolumn{5}{c}{ Eluting pump } \\
\hline Step & Time (s) & Flow (ml/min) & A & B & C & D & Flow (ml/min) & A & B \\
1 & 30 & 1.5 & & & & 100 & 0.5 & 100 & - \\
2 & 90 & 0.1 & 100 & & & & 0.4 & 100 & - \\
3 & 15 & 2.0 & & & 100 & & 0.5 & 75 & 25 \\
4 & 15 & 2.0 & & & & 100 & 0.5 & 50 & 50 \\
5 & 15 & 2.0 & & & 100 & & 0.5 & 40 & 60 \\
6 & 15 & 2.0 & & & & 100 & 0.5 & 30 & 70 \\
7 & 15 & 2.0 & 100 & & & & 0.5 & 20 & 80 \\
8 & 15 & 2.0 & & 100 & & & 0.5 & - & 100 \\
9 & 60 & 2.0 & & 100 & & & 0.5 & - & 100 \\
10 & 30 & 2.0 & & & 100 & & 0.5 & 100 & - \\
11 & 30 & 0.5 & & & 100 & & 0.5 & 100 & - \\
12 & 120 & 1.5 & & & & 100 & 0.5 & 100 & - \\
\hline
\end{tabular}

zation of the SRM parameters was performed by direct infusion of the standards using positive electrospray ionisation. Collision-induced dissociation (CID) mass spectra were recorded for each analyte and the optimum collision energies determined for the selected ion transitions. Two transitions were monitored for each analyte as represented in Table 2.

Method validation

The method was validated based on the criteria for screening methods as described by the EU (Commission Decision 2002/657/EC [13]). For screening methods it is necessary to determine the beta-error of the screening method. In this study, the standard mixture of veterinary drugs was added to three different milk samples at four concentration levels viz. 50-100-250-500 $\mu \mathrm{g} / \mathrm{L}$. All analysis were performed in triplicate. In this way a total number of $4 \times 3 \times 3$ samples are analysed per analyte. Samples were analysed together with a calibration curve of neat standard solutions in the concentration range of 5-500 $\mu \mathrm{g} / \mathrm{L}$. When all the fortified samples are screening 'positive', the method will be accepted. From this experiment, the linearity, repeatability, limit of detection, carry-over and matrix effect were also determined. Furthermore, the robustness of the method was tested by the analysis of samples with different fat content.

The linearity of the analytical method was studied by preparing calibration curves at different concentration levels. The calibration curves were constructed by means of plotting the detection response of the matrix matched standard solutions (spiked samples) versus the concentrations by means of regression analysis. From these data, the regression coefficients $\left(r^{2}\right)$ of the calibration curves were calculated. Criterion for good linearity: $r^{2}>0.99$.

The repeatability was determined by the analysis of three replicates of each test sample. The repeatability-expressed as RSD (\%) —was automatically calculated using the quantification software. Criterion for good repeatability was set at $\mathrm{RSD}<15 \%$.

Limits of detection (LODs) were set at those concentration levels in matrix showing $S / N$ of 6 .

For automatic sample pre-treatment, it is important to know if there is carry-over of the analyte from one injection to the next. By analysing spiked samples, it was possible to test the carry-over. First, the spike sample was injected and directly after that blank from the results the carry-over percentages were determined.
Table 2 Selected ion transitions for the monitoring of the different analytes by TFC-LC(ESI)-MS/MS

\begin{tabular}{lccccc}
\hline Analyte & Precursor ion $(\mathrm{m} / \mathrm{z})$ & Product 1 & $\mathrm{CE}(\mathrm{V})$ & Product 2 & CE (V) \\
\hline Albendazole & 266.1 & 234.0 & 15 & 191.0 & 31 \\
Sulphamethazine & 279.1 & 124.2 & 14 & 108.0 & 16 \\
Phenylbutazone & 309.2 & 211.3 & 16 & 188.3 & 15 \\
Difloxacin & 400.1 & 356.1 & 20 & 299.1 & 27 \\
Spiramycin & 422.0 & 174.0 & 35 & 350.5 & 12 \\
Tetracycline & 445.5 & 410.0 & 17 & 427.0 & 6 \\
Oxytetracycline & 461.1 & 426.0 & 19 & 201.0 & 36 \\
Salinomycin-Na & 773.4 & 265.4 & 50 & 432.0 & 44 \\
\hline
\end{tabular}


The matrix effect was studied by comparing the results obtained for spiked milk samples and direct injection of the standard solutions with the same concentrations of veterinary drugs. A mixture of standards at $100 \mu \mathrm{g} / \mathrm{L}$ level was prepared in methanol and analysed by TFC-LC-MS/MS. The area counts for each analyte were then compared with the one obtained on the analysis of the same mixture in matrix (milk). Two different milk samples were studied and referenced as A (low fat milk) and B (whole-fat milk).

The robustness of the developed method was tested by comparing the results obtained for different milk samples with low, medium and high fat content.

\section{Application}

The method was applied to the analysis of 12 raw milk samples. For testing purposes, some of the milk samples were fortified with a veterinary drug. This set of samples was analysed using the developed TFC-LC-MS/MS method. All samples were previously analysed by the authors using a normal routine LC-MS/MS method with offline sample preparation [3] and were found negative. Negative means less than the EU MRL for the specific compounds in milk. For albendazole, sulphamethazine, tetracycline and oxytetracycline negative means $<100 \mu \mathrm{g} / \mathrm{L}$, for spiramycine $<200 \mu \mathrm{g} / \mathrm{L}$ and for the non-MRL substances phenylbutazone, difloxacin, salinomycin-Na, negative means $<10 \mu \mathrm{g} / \mathrm{L}$.

The routine analysis of the raw milk samples by TFCLC-MS/MS was performed by a technician not aware of the concentration of drugs residues present in the samples. In this way, the method was tested for false positive and negative results.

\section{Results and discussion}

\section{Method development}

Four steps were considered when developing the TFCmethod. The first consisted of a loading step and involved the loading of the sample and sufficient washing of the TFC column to remove the matrix. Water containing $2 \%$ acetonitrile and $0.1 \%$ ammonium hydroxide proved to be suitable for removing the matrix from the TFC column while retaining the analytes of interest. The second step was the transfer step in which the analytes of interest were desorbed from the TFC column onto the analytical column. At this stage, the TFC flow rate was decreased before its inline mixing with the low organic content delivered from elution pump of the analytical column. Lowering the organic content prevented the dispersion of the analytes in the analytical column and consequent peak broadening. Methanol proved to be the most suitable solvent for complete recovery of the analytes from the TFC column. The third step is the actual chromatographic separation on the analytical LC column using an elution gradient. The fourth and last step was the equilibration in which the initial conditions were set for the next run. Note that equilibration and loading of the TFC column for the next run can already start before the end of the analytical separation. To avoid carry-over, the composition of the conditioning solution for the TFC column was also tested. A mixture of isopropanol and acetone (1/1) was selected as most suitable.

Method validation

All spiked samples were found positive in the screening analysis. That means that no false negative results were obtained for the analysis of all the spiked samples. The samples analysed without the addition of veterinary drugs were all screened negative. Table 3 presents the results obtained for the linearity and repeatability. The linearity was found to be good with the exception of spiramycin low fat, and oxytetracycline whole milk. On both cases, the correlation coefficient was below 0.99. Calibration curves constructed based on neat standard solutions appear to be linear within the range of $5-500 \mu \mathrm{g} / \mathrm{L}$. For repeatability, RSD below $15 \%$ were obtained for the majority of the analysis, with sulphamethazine and difloxacin for whole milk and at the highest level $(500 \mu \mathrm{g} / \mathrm{L})$, as the exceptions. This is an indication that the method developed is able to successfully separate the matrix from the analytes of interest. The same was confirmed by the analysis of a blank milk sample. No major interferences were found at the analytes' retention times.

A representative SRM chromatogram is shown in Fig. 2. The concentration level selected was $100 \mu \mathrm{g} / \mathrm{L}$. Unambiguous peaks were observed for each transition monitored (only one SRM transition shown) demonstrating the good performance of the extraction procedure. However, it should be kept in mind that the development of multiresidue methods using mass spectrometric detection often leads to instrumental parameter settings that are adequate for the determination of all analytes but are not necessarily optimal for individual analytes. Furthermore, when dealing with on-line sample preparation methodologies like turbulent flow chromatography, factors such as the $\mathrm{pH}$ during extraction may be crucial for an efficient extraction procedure. Therefore, compromises need to be made, but in such a way that the desired analytical requirements as linearity, repeatability and limits of detection will be met for all the compounds present in the method.

Since the peak intensity of an LC-MS/MS signal is related to the ionisation efficiency of the individual analyte while passing from the solution phase to the gas phase, the ionisation efficiency of the targeted compounds plays an 
Table 3 The variation in linearity $\left(r^{2}\right)$, slope, intercept and repeatability (RSD\%) on the TFC-LC-(ESI)-MS/MS analysis of different fat content milk samples $(n=3)$

\begin{tabular}{|c|c|c|c|c|c|c|c|c|c|c|c|c|c|}
\hline \multirow{2}{*}{\multicolumn{2}{|c|}{$\begin{array}{c}\text { Milk Sample } \\
\text { Fortification Level }(\mu \mathrm{g} / \mathrm{L})\end{array}$}} & \multicolumn{4}{|c|}{ Non-Fat } & \multicolumn{4}{|c|}{ Low Fat $(2 \%)$} & \multicolumn{4}{|c|}{ Whole Milk } \\
\hline & & 50 & 100 & 250 & 500 & 50 & 100 & 250 & 500 & 50 & 100 & 250 & 500 \\
\hline \multirow{2}{*}{ Albendazole } & $r^{2}$ & \multicolumn{4}{|c|}{$\begin{array}{c}\mathrm{y}=-199899+32597 \mathrm{x} \\
0.9984\end{array}$} & \multicolumn{4}{|c|}{$\begin{array}{c}y=-312516+36317 x \\
0.9967\end{array}$} & \multicolumn{4}{|c|}{$\begin{array}{c}\mathrm{y}=-454265+36722 \mathrm{x} \\
0.9928\end{array}$} \\
\hline & $\begin{array}{l}\mathrm{RSD} \\
(\%)\end{array}$ & 1.6 & 1.7 & 1.7 & 2.7 & 6.3 & 3.2 & 3.6 & 4.2 & 2.6 & 6.2 & 1.2 & 2.9 \\
\hline \multirow{2}{*}{ Sulphamethazine } & $r^{2}$ & \multicolumn{4}{|c|}{$\begin{array}{c}\mathrm{y}=291+580 \mathrm{x} \\
0.9964\end{array}$} & \multicolumn{4}{|c|}{$\begin{array}{l}y=5511+529 x \\
0.9908\end{array}$} & \multicolumn{4}{|c|}{$\begin{array}{c}\mathrm{y}=2153+638 \mathrm{x} \\
0.9970\end{array}$} \\
\hline & $\begin{array}{c}\mathrm{RSD} \\
(\%)\end{array}$ & 2.4 & 7.2 & 4.9 & 2.4 & 6.6 & 14.5 & 5.2 & 5.6 & 8.9 & 1.2 & 5.1 & 18.4 \\
\hline \multirow{2}{*}{ Phenylbutazone } & $r^{2}$ & \multicolumn{4}{|c|}{$\begin{array}{c}y=-211312+6023.8 x \\
0.9947\end{array}$} & \multicolumn{4}{|c|}{$\begin{array}{c}\mathrm{y}=-305494+7911 \mathrm{x} \\
0.9922\end{array}$} & \multicolumn{4}{|c|}{$\begin{array}{c}y=-420552+7391 x \\
0.9963\end{array}$} \\
\hline & $\begin{array}{c}\mathrm{RSD} \\
(\%)\end{array}$ & 2.9 & 3.3 & 0.8 & 2.6 & 8.1 & 4.1 & 4.9 & 3.1 & 0.6 & 0.9 & 0.7 & 0.3 \\
\hline \multirow{2}{*}{ Difloxacin } & $r^{2}$ & \multicolumn{4}{|c|}{$\begin{array}{c}y=444653+23806 x \\
0.9958\end{array}$} & \multicolumn{4}{|c|}{$\begin{array}{c}\mathrm{y}=309858+24725 \mathrm{x} \\
0.9907\end{array}$} & \multicolumn{4}{|c|}{$\begin{array}{c}\mathrm{y}=173467+27985 \mathrm{x} \\
0.9968\end{array}$} \\
\hline & $\begin{array}{l}\mathrm{RSD} \\
(\%)\end{array}$ & 12.2 & 4.3 & 6.0 & 2.4 & 10.8 & 4.6 & 2.7 & 5.5 & 2.6 & 6.1 & 5.1 & 15.4 \\
\hline \multirow{2}{*}{ Spiramycin } & $r^{2}$ & \multicolumn{4}{|c|}{$\begin{array}{c}\mathrm{y}=19898+333 \mathrm{x} \\
0.9920\end{array}$} & \multicolumn{4}{|c|}{$\begin{array}{c}y=6170+418 x \\
0.9740\end{array}$} & \multicolumn{4}{|c|}{$\begin{array}{c}\mathrm{y}=5433+476 \mathrm{x} \\
0.9951\end{array}$} \\
\hline & $\begin{array}{c}\mathrm{RSD} \\
(\%)\end{array}$ & 11.1 & 11.8 & 8.4 & 4.1 & 10.9 & 4.0 & 10.0 & 9.4 & 13.3 & 6.5 & 5.2 & 0.2 \\
\hline \multirow{2}{*}{ Tetracycline } & $r^{2}$ & \multicolumn{4}{|c|}{$y=-15636+3095 x$} & \multicolumn{4}{|c|}{$\begin{array}{c}y=-31979+3179 x \\
0.9948\end{array}$} & \multicolumn{4}{|c|}{$\begin{array}{c}y=-71774+3787 x \\
0.9903\end{array}$} \\
\hline & $\begin{array}{l}\text { RSD } \\
(\%)\end{array}$ & 6.2 & 6.4 & 5.4 & 3.7 & 7.3 & 4.8 & 5.9 & 4.5 & 4.1 & 9.5 & 6.5 & 5.5 \\
\hline \multirow{2}{*}{ Oxytetracycline } & $\mathrm{r}^{2}$ & \multicolumn{4}{|c|}{$\begin{array}{c}y=-4096+1135 x \\
0.9947\end{array}$} & \multicolumn{4}{|c|}{$\begin{array}{c}y=-2099+1192 x \\
0.9922\end{array}$} & \multicolumn{4}{|c|}{$\begin{array}{c}\mathrm{y}=-23879+1439 \mathrm{x} \\
0.9663\end{array}$} \\
\hline & $\begin{array}{c}\text { RSD } \\
(\%)\end{array}$ & 2.9 & 3.3 & 0.8 & 2.6 & 8.1 & 4.1 & 4.9 & 3.1 & 0.6 & 0.9 & 0.7 & 0.3 \\
\hline \multirow{2}{*}{ Salinomycin-Na } & $r^{2}$ & $\mathrm{y}=$ & $\begin{array}{r}5538 \\
0.5\end{array}$ & $\begin{array}{l}+123 \\
93 \\
9\end{array}$ & $84 \mathrm{x}$ & $\mathrm{y}=$ & $\begin{array}{r}9708 \\
0 .\end{array}$ & $\begin{array}{l}+125 \\
66\end{array}$ & $8 x$ & $\mathrm{y}=$ & $\begin{array}{r}71219 \\
0.9\end{array}$ & 141 & \\
\hline & $\begin{array}{c}\mathrm{RSD} \\
(\%)\end{array}$ & 1.2 & 0.7 & 1.2 & 1.5 & 1.5 & 0.8 & 3.1 & 0.4 & 2.8 & 3.1 & 1.3 & 1.2 \\
\hline
\end{tabular}

$\mathrm{RSD} \%$ of the repeatability for the specific sample type/concentration level $(n=3)$

essential role in defining the accuracy of the measurements. The ionisation efficiency can be either suppressed or enhanced due to the presence of other materials, often related to the matrix. The co-elution of other compounds, either originating from the matrix or simply other analytes present in the sample, can influence the response and therefore interfere with the quantification. Electrospray ionisation (ESI) is reported to be more likely impacted by these factors than atmospheric pressure chemical ionisation (APCI). The main cause for ion suppression is a change in the spray droplet solution properties caused by the presence of non-volatile or less volatile solutes. These compounds can change the efficiency of droplet formation or droplet evaporation, which in turn affects the amount of charged ion in the gas phase that ultimately reaches the detector. One objective of this study was to evaluate how co-eluting matrix interferences would influence the relative response for our selected analytes.

For this study, the standards and the samples are treated exactly the same namely dilution, centrifugation, injection.
Therefore, the matrix effects are representative of recovery for this method. Table 4 presents the results of matrix effects (ion suppression or enhancement), LODs and carryover. It can be seen that ion suppression was dominant, but also that signal enhancement was found for difloxacin and tetracycline in milk samples A and B and for oxytetracycline in milk sample A (low fat) only. If a comparison is made between the two samples, it can be concluded that the change in the response (either suppression or enhancement) is similar in both samples of milk. This indicates that the composition of the samples is probably similar and that the different fat content does not introduce additional variability into the analysis. Phenylbutazone and difloxacin are the exception since the differences in signal suppression and enhancement for the two samples of milk are relatively large compared to the other compounds.

Albendazole is the analyte most affected by matrix effects and also the one showing the highest carry-over value. However, $1.2 \%$ is still below the lowest point of the selected calibration range. Albendazole also presented the 
Fig. 2 Representative SRM chromatograms of a non-fat milk sample spiked with the mixture of antibiotics standards at $100 \mu \mathrm{g} / \mathrm{L}$ level and analysed by TFC-LC-(ESI)-MS/MS. See Table 2 for details

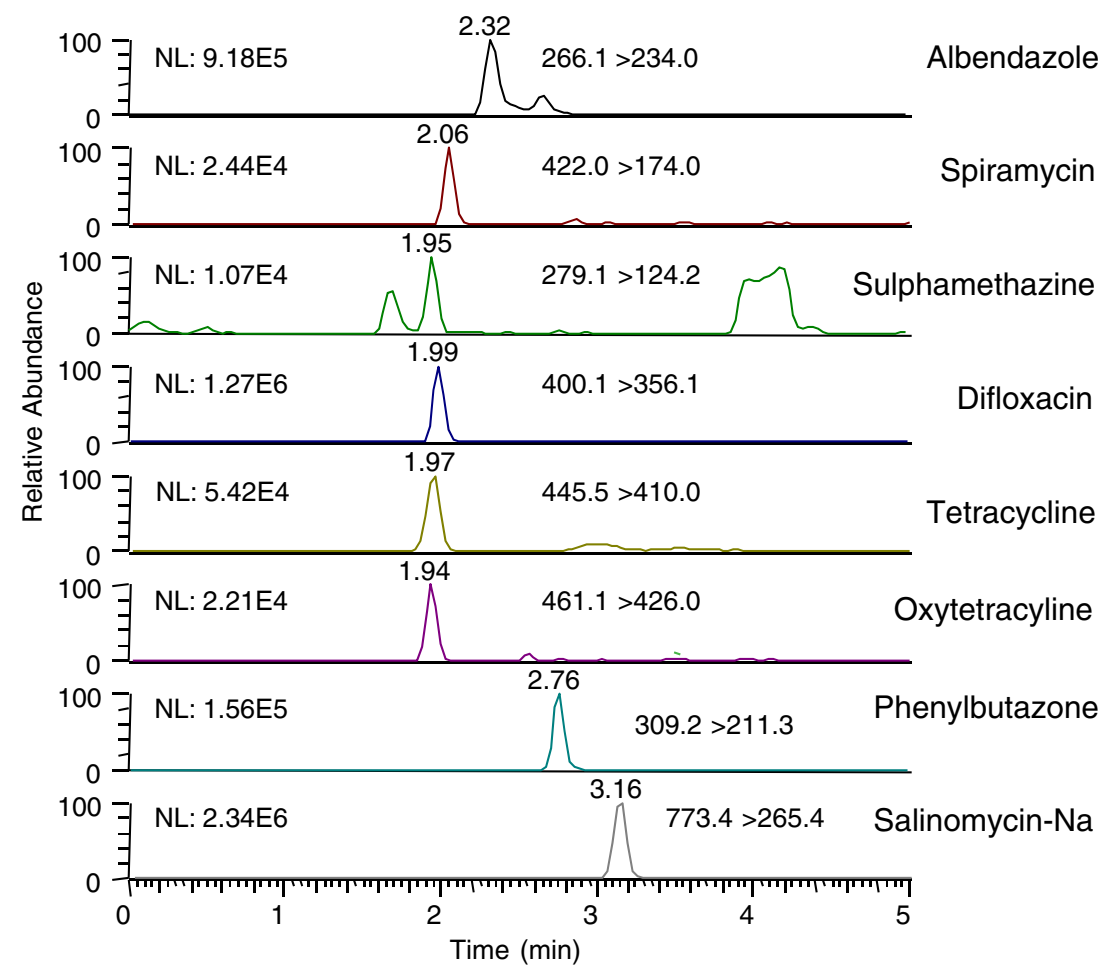

lowest limit of detection, $0.4 \mu \mathrm{g} / \mathrm{L}$. On the contrary, tetracycline was the least affected by the presence of the matrix. It is expected that the conditions selected in this method, e.g., $\mathrm{pH}$ used on the extraction procedure, favour certain groups in relation to others, due to the different chemical properties of each analyte. The method seems to be closer to optimum to compounds belonging to the tetracycline family, macrolides and even ionophores than to the benzimidazoles.

Aguilera-Luiz et al. [6] also observed matrix effects by the analysis of veterinary drug in milk. For some benzimidazoles and quinolones, they observed enhance- ment effects and for the levamisol and abamectin significant ion suppression effects. However, they did not quantify these effects. Furthermore, they concluded-like in the present study - that there was no significant influence of the sample composition. In other words, almost no differences in matrix effects between non-fat, whole-fat and medium-fat milk samples.

The LODs ranged from 0.4 to $5.2 \mu \mathrm{g} / \mathrm{L}$ (see Table 4 ) and are far below the MRLs. According to new EU guidelines for the validation of a screening method (CRL document of 20/1/2010 Guidelines for the validation of screening methods for residues of veterinary medicines-initial
Table 4 Relative responses (\%), LODs and carry-over at $100 \mu \mathrm{g} / \mathrm{L}$ level

\footnotetext{
${ }^{\text {a }}$ Relative response $(\%)=($ Area milk sample/Area neat standard -1$) \times 100$

${ }^{\mathrm{b}}$ Limit of detection in matrix

${ }^{\mathrm{c}}$ Carry-over $(\%)=($ Area blank $/$ Area milk sample (level $500 \mu \mathrm{g} / \mathrm{L})) \times 100$
}

\begin{tabular}{lcccc}
\hline Analyte & \multicolumn{2}{l}{ Relative response ${ }^{\mathrm{a}}(\%)$} & LOD $^{\mathrm{b}}(\mu \mathrm{g} / \mathrm{L})$ & Carry-over ${ }^{\mathrm{c}}(\%)$ \\
\cline { 2 - 4 } & $\mathrm{A}=$ Low fat milk & $\mathrm{B}=$ Whole fat milk & & \\
\hline Albendazole & -82 & -85 & 0.4 & 1.2 \\
Sulphamethazine & -57 & -59 & 1.6 & 0 \\
Phenylbutazone & -69 & -25 & 1.9 & 0 \\
Difloxacin & 70 & 40 & 1.7 & 0.6 \\
Spiramycin & -28 & -37 & 5.2 & 0 \\
Tetracycline & 8 & 8 & 2.4 & 0 \\
Oxytetracycline & 31 & -5 & 3.0 & 0 \\
Salinomycin-Na & -19 & -31 & 0.7 & 0.2 \\
\hline
\end{tabular}


Table 5 Testing the method as a routine procedure

\begin{tabular}{lll}
\hline Sample & Analytes added & Analysis by TFC-LC-(ESI)-MS/MS \\
\hline 01 & None & Negative \\
02 & None & Negative \\
03 & None & Negative \\
04 & None & Negative \\
05 & None & Negative \\
06 & None & Negative \\
07 & None & Negative \\
08 & None & Negative \\
09 & None & Negative \\
10 & None & Oxytetracycline $5 \mu \mathrm{g} / \mathrm{L}$ \\
11 & Oxytetracycline $200 \mu \mathrm{g} / \mathrm{L}$ & Oxytetracycline $1 \quad \mathrm{mg} / \mathrm{L} \mathrm{Tetracycline} 5 \quad \mu \mathrm{g} / \mathrm{L}$ \\
12 & Sulphamethazine $200 \mu \mathrm{g} / \mathrm{L}$ & Sulphamethazine $200 \mu \mathrm{g} / \mathrm{L}$ \\
\hline
\end{tabular}

validation and transfer ${ }^{1}$ ), a cut-off level has to be established for each analyte/matrix combination. A cut-off level is defined as the level which indicates that a confirmatory test has to be performed. For non-MRL substances the cut-off have to be as low as possible preferably around the LOD and for MRL substances the cut-off has to be preferably around $1 / 2 \mathrm{MRL}$. With the very low LODs obtained with the present method, these required cut-off levels were obtained.

While some matrix effects remain for all the analytes the developed method has proved to be linear, repeatable and low limits of detection were obtained. Also, the results appear to be consistent for the different samples studied indicating that similar results can be obtained when samples originating from different sources are analysed. Carry-over was minimal for most of the analytes which indicates that the solutions involved in the cleaning process are efficient on removing any residues from the system between injections.

\section{Application}

The results of the analysis of the series of raw milk samples are presented in Table 5.

From the results, it is concluded that no false negatives or positives were found. However, lower amounts of tetracyclines were found in samples 10 and 11. These analytes were not added to the sample. The amount detected is close to their limit of detection and one can question if these analytes were already present in the blank sample used. The concentrations detected are far below the MRLs and so these concentrations will not be picked up in regular LC-MS/MS method used for monitoring purposes. While the concentration found for sulphamethazine in

\footnotetext{
${ }^{1}$ See http://ec.europa.eu/food/food/chemicalsafety/residues/Guideline Validation_Screening_en.pdf.
}

sample 12 is correct, the oxytetracycline concentration found in sample 11 is five times higher than expected. Signal enhancement due to matrix components alone cannot account for this overestimation and better quantification results may have been obtained if an internal standard is used. Nevertheless, the method was able to detect all the compounds present in the blind samples, proving to be a fast and efficient screening tool. In practical applications, the three positive samples from this screening would have been re-analysed for confirmation purposes, thus confirming true findings and removing any false positives.

\section{Conclusion}

A new method for the automated multi-residue screening of antibiotics in milk is proposed. It combines turbulent flow chromatography for analyte extraction and purification with LC-MS/MS for analytical separation and detection of the analytes. The advantages of this method are the reduced sample preparation time and higher sample throughput.

Albendazole, sulphamethazine, phenylbutazone, difloxacin, spiramycine, tetracycline, oxytetracycline, and salinomycin-Na were isolated, detected and quantified in milk by TFC-LC-ESI-MS/MS. Sample pre-treatment was limited to diluting and centrifugation. Even without internal standards, good linearity was obtained in the concentration range of 50 to $500 \mu \mathrm{g} / \mathrm{L}$ (for neat standard in the range $5-500 \mu \mathrm{g} / \mathrm{L})$ as well as repeatability (RSDs $<15 \%$ exception: sulphamethazine and difloxacin at $500 \mu \mathrm{g} / \mathrm{L}<20 \%$ ). The limits of detection were between 0.1 and $5.2 \mu \mathrm{g} / \mathrm{L}$. The limits of detection found were far below the MRLs of the tested analytes. While matrix effects (ion suppression or enhancement) were obtained for all the analytes the method has proved to be useful for screening purposes because of its sensitivity. The results appear to be consistent for the 
different samples tested indicating that similar results can be obtained when samples originating from different sources are analysed. Carry-over was minimal for most of the analytes which indicates that the solutions involved in the cleaning process are efficient on removing any residues from the system between injections.

The developed TFC-LC-MS/MS method is a quick and sensitive screening method for automated multi-residue screening of veterinary drugs in milk.

Acknowledgement This project was financially supported by the Dutch Ministry of Agriculture, Nature and Food Quality (project 7261201).

Open Access This article is distributed under the terms of the Creative Commons Attribution Noncommercial License which permits any noncommercial use, distribution, and reproduction in any medium, provided the original author(s) and source are credited.

\section{References}

1. Official Journal of the European Union, L224 18 August 1990, Council Regulation 2377/90/EC of 26 June 1990 laying down a Community procedure for the establishment of maximum residue limits of veterinary medicinal products in foodstuffs of animal origin, Brussels, Belgium, 1990

2. Official Journal of the European Communities L224, of 18 August 1990, Council Regulation 2377/90/EC; consolidated version of the Annexes I to IV updated up to 22.12.2004 obtained from www.emea.eu.int

3. Stolker AAM, Brinkman UATh (2005) J Chromatogr A 1067:1553

4. Turnipseed SB, Andersen WC, Karbiwnyk CM, Madson MR, Miller KE (2008) Rapid Commun Mass Spectrom 22:14671480

5. Gaugain-Juhel M, Delépine B, Gautier S, Fourmond MP, Gaudin V, Hurtaud-Pessel D, Verdon E, Sanders P (2009) Food Addit Contam 11:1459-1471

6. Aguilera-Luiz MM, Vidal JLM, Romero-González R, Frenich AG (2008) J Chromatogr A 1205:10-16

7. Kinsella B, O'Mahony J, Malone E, Moloney M, Cantwell H, Furey A, Danaher M (2009) J Chromatogr A 1216:7977-8015

8. Quinn HM, Takarewski JJ (1997) Int. Pat., WO 97/16724, p 91, chapter 4

9. Edge T (2002) Chromatogr Sep Technol 23:10-16

10. Xu Y, Willson KJ, Musson DG (2008) J Chromatogr B 863:64-73

11. Mottier P, Hammel YA, Gremaud E, Philippe AG (2008) J Agric Food Chem 56:35-43

12. Krebber R, Hoffend FJ, Ruttman F (2009) Anal Chim Acta 637:208-213

13. Official Journal of the European Communities L221, 8-36. Commission Decision (2002/657/EC) of 12 August 2002, Brussels, Belgium, 2002 